\title{
Using X-ray footprinting to investigate protein interactions and conformation
}

Corie Y. Ralston and Sayan Gupta

Molecular Biophysics and Integrated Bioimaging Division, Lawrence Berkeley National Laboratory, Berkeley, CA 94720; cyralston@lbl.gov and sayangupta@lbl.gov

The use of X-ray footprinting mass spectrometry (XFMS) to investigate structural features and conformational changes of macromolecules in the solution state has grown substantially in the past decade and has been successfully applied to systems ranging from single domain proteins to in vivo ribonucleoprotein assemblies. The method is highly complementary to the more widely used structural elucidation techniques for biological macromolecules such as X-ray diffraction, HDX, and cryo-electron microscopy. XFMS is an in situ hydroxyl radical $(\cdot \mathrm{OH})$ labeling method; X-ray irradiation dissociates solvent water to produce hydroxyl radicals, which covalently modify side chains which are solvent accessible. More specifically, residues which are in proximity to water molecules (either bulk or bound) are modified to a greater extent than residues which are not in proximity to water. Because liquid chromatography-mass spectrometry is then used to analyze the stable covalent modifications produced, the data provide a "water map" at the single residue level, which is then used to determine sample conformation. In this talk, I will describe the XFMS method, its advantages and disadvantages relative to other methods, and some recent exciting examples of structural information obtained on protein systems using the method. 